\title{
Association between spinal alignment and biochemical composition of lumbar intervertebral discs assessed by quantitative magnetic resonance imaging
}

\author{
Rafael Menezes-Reis ${ }^{1,2}$, Carlos E. Garrido Salmon ${ }^{3}$, Gustavo P. Bonugli ${ }^{1}$, Debora Mazoroski ${ }^{1}$, \\ Leonor G. Savarese ${ }^{1}$, Carlos Fernando P. S. Herrero ${ }^{1}$, Helton L. A. Defino ${ }^{1}$, \\ Marcello Henrique Nogueira-Barbosa ${ }^{1}$
}

${ }^{1}$ Ribeirão Preto Medical School - University of São Paulo, Ribeirão Preto, SP, Brazil; ${ }^{2}$ Federal University of Amazonas, Institute of Health and Biotechnology, Coari, AM, Brazil; ${ }^{3}$ Ribeirão Preto Philosophy and Sciences School - University of São Paulo, Ribeirão Preto, SP, Brazil

Correspondence to: Rafael Menezes-Reis. Universidade Federal do Amazonas - Instituto de Saúde e Biotecnologia, Estrada Coari/Mamiá, 305, Coari, Amazonas, Brazil. Email: rafaelmenezesreis@gmail.com.

\begin{abstract}
Background: To evaluate potential associations between spinopelvic parameters and the biochemical composition of lumbar intervertebral discs using quantitative magnetic resonance imaging in asymptomatic young adults.

Methods: Our study group comprised 93 asymptomatic volunteers aged 20-40 years (49 women and 44 men). Lumbar spine T2-weighted images and T2 relaxometry were acquired on a 1.5T MRI scanner. Spinopelvic parameters including sacral slope, pelvic tilt, pelvic incidence, lumbar lordosis, thoracic kyphosis, thoracolumbar alignment, sagittal vertical axis, spinosacral angle, C2 pelvic angle, and T1S1 and L1S1 length were measured on panoramic spine radiographs.

Results: Lumbar lordosis decrease correlates with discrete dehydration of nucleus pulposus at all lumbar levels. Also low values of sacral slope, pelvic tilt, pelvic incidence, thoracic kyphosis and spinosacral angle were associated with decrease of T2 relaxation times on annulus fibrosus.
\end{abstract}

Conclusions: In conclusion, spinopelvic parameters presented a discrete association with lumbar disc composition and water content.

Keywords: Intervertebral disc; spine; posture; magnetic resonance imaging; lumbosacral region

Submitted Jan 07, 2020. Accepted for publication Jan 07, 2021.

doi: $10.21037 /$ qims-20-40

View this article at: http://dx.doi.org/10.21037/qims-20-40

\section{Introduction}

The intervertebral discs (IVDs) are crucial for spine balance $(1,2)$, and IVD degeneration is associated with instability, deformities, back pain and sensorimotor disabilities (3). Disc degeneration occurs with loss of proteoglycans and type II collagen in the extracellular matrix of the nucleus pulposus (NP) and annulus fibrosus (AF) derangement (1).

In asymptomatic subjects, the prevalence of disc degeneration can range from $30 \%-95 \%$ depending on the age of the study participants (4). Lumbar disc degeneration was present in over $30 \%$ of asymptomatic subjects and more than $50 \%$ of symptomatic subjects older than 50 years (4). Disc degeneration is not always associated with back pain in young patients (5). Due to the high prevalence of IVD degeneration in the general population, possible risk factors for the development of lumbar disc degeneration should be examined (6).

Mechanical stress could accelerate the development of disc degeneration and herniation (7). Sagittal balance is associated with the spine biomechanics and influences degenerative diseases $(8,9)$. Chronic low back pain and spinal degenerative diseases are associated with an anterior 
Table 1 List of acronyms and meanings related to the study

\begin{tabular}{ll}
\hline Name & Acronym \\
\hline NP & Nucleus pulposus \\
AAF & Anterior annulus fibrosus \\
SAF & Posterior annulus fibrosus \\
PT & Sacral slope \\
PI & Pelvic tilt \\
LL & Pelvic incidence \\
TK & Lumbar lordosis \\
TL & Thoracic kyphosis \\
CPA & Thoracolumbar alignment \\
SVA & C2 Pelvic angle \\
SSA & Sagittal vertical axis \\
T1S1 & Spinosacral angle \\
L1S1 & T1 vertebra to Sacrum length \\
\hline
\end{tabular}

sagittal imbalance, loss of lumbar lordosis and increased pelvic tilt (10). Although a sagittal imbalance has being associated with disc disorders, little is known about the influence of the mechanical loads generated by the sagittal balance on the human disc biochemical composition. The axial mechanical load affects proteoglycan and collagen synthesis and disc hydration, according to experimental and cadaveric studies (11).

Quantitative magnetic resonance imaging (MRI) of IVDs allows in vivo detection of decreased proteoglycan, water and glycosaminoglycan levels and increased collagen content related to degeneration process (12). T2 and T1 $\rho$ relaxometry are quantitative techniques that have been validated for the in vivo detection of early degenerative processes $(13,14)$. The $\mathrm{T} 2$ relaxometry values correlate strongly with the disc NP water content and to a lesser extent with the proteoglycans, whereas these values are inversely related to the collagen content (15). Disc degeneration and ageing may be characterized by a progressive decrease of $\mathrm{T} 2$ relaxation time related to dehydration, loss of proteoglycans and collagen substitutions (13-15).

Our hypothesis is that the mechanical loads related to different spinal sagittal balance types or parameters may be associated with early changes in intervertebral discs. Therefore, we evaluated possible correlations between the spinal sagittal balance parameters and the lumbar IVD biochemical composition measured by $\mathrm{T} 2$ relaxometry.

\section{Methods}

\section{Sample}

This study was an observational, prospective and crosssectional. The volunteer's recruitment occurred through invitation and divulgation on posters in the city and social networks, and the subjects were screened according to selection criteria. The inclusion criteria were as follows: 20 to 40 years old, Oswestry Disability Index score of less than 10 and a classification of sedentary or irregularly active according to the International Physical Activity Questionnaire. We excluded volunteers with persistent low back pain for more than three months, history of radiculopathy, neuromotor disorders, facet joint or hip osteoarthritis, previous spine or hip surgery, scoliosis, spine or hip fracture, osteoporosis and pregnancy.

Our final sample comprised 93 young adult asymptomatic volunteers (49 women and 44 men).

\section{Spinopelvic balance evaluation}

Each volunteer underwent a panoramic radiograph acquired with a CR Long Length Vertical Imaging System (Kodak Direct View, Carestream Health, Rochester, NY, USA). The digital images were acquired in the lateral view with a focus-film distance of $2 \mathrm{~m}$. The subjects were placed in standing position with their arms supported on a stand, shoulders at $30^{\circ}$ of flexion and slightly flexed elbows according to previous literature (16).

Surgimap $^{\circledR}$ software (Nemaris Inc., New York, NY, USA) was used to measure the spinopelvic parameters (Table 1). A researcher with seven years of experience in spinal imaging measurements was responsible for the manual measurements. The following parameters were evaluated (Figure 1): sacral slope (SS), pelvic tilt (PT), pelvic incidence (PI), lumbar lordosis (LL), thoracic kyphosis (TK), thoracolumbar alignment (TL), sagittal vertical axis (SVA), spinosacral angle (SSA), C2 pelvic angle (CPA), and T1S1 and L1S1 length. Surgimap ${ }^{\circledR}$ was used to mark the femoral heads contours and the lines adjacent to the vertebral body of the sacral endplate, upper endplate of L1, upper endplate of T1 and lower endplate of C2. The software automatically calculated the spinopelvic parameters and vertebral curvatures. A second examiner, blind to the first assessment, performed the same measurements on all sample and with 

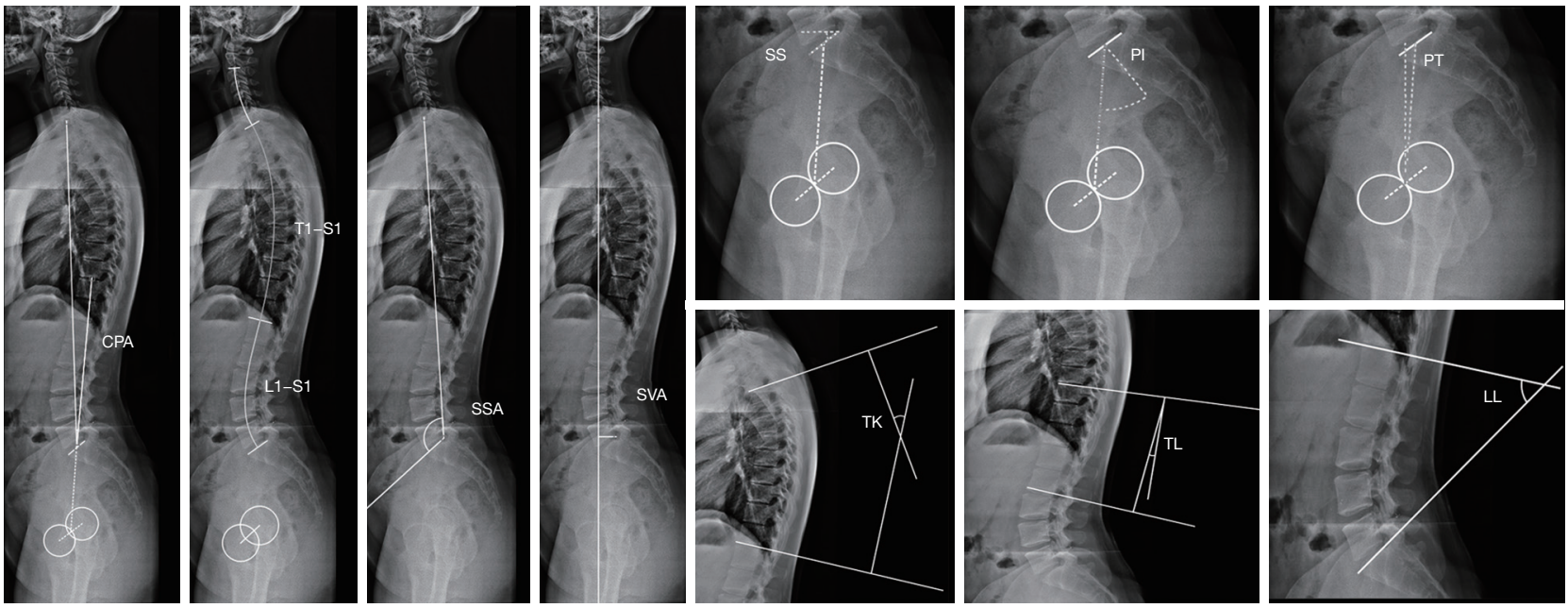

Figure 1 Panoramic radiograph of a female volunteer illustrating the spinopelvic parameter measurements. The following parameters were measured: CPA, C2 pelvic angle; T1S1 length and L1S1 length; SSA, spinosacral angle; SVA, sagittal vertical axis; SS, sacral slope; PT, pelvic tilt; PI, pelvic incidence; LL, lumbar lordosis; TK, thoracic kyphosis; TL, thoracolumbar alignment.

same methods to verify inter-observer agreement. The values used for statistical analysis were the first examiner results.

We classified the volunteers according to the Roussouly classification system (17) to assess potential significant differences in the lumbar IVD biochemical composition between the different postural subtypes (Figure 2). Two researchers with experience with posture analysis classified the volunteers in consensus. For doubtful cases, a third observer, a senior radiologist with 20 years of experience on musculoskeletal radiology, defined the final classification.

\section{Disc composition evaluation}

We evaluated lumbar IVD composition using $1.5 \mathrm{~T}$ MRI scanner (Achieva; Philips Healthcare, Best, The Netherlands) with a 16-channel spinal coil. The volunteers were evaluated in the supine position with extended legs. Prior to the MRI acquisition, the volunteers rested in a seated position for 1 hour. To avoid possible physiologic daily variations in disc water content, MRIs were acquired in the afternoon.

All images of the lumbar spine were acquired in the sagittal plane using the following parameters: field of view $=22 \times 22 \mathrm{~cm}$; thickness $=4 \mathrm{~mm}$; number of slices $=16$; and matrix $=256 \times 256$. A $2 \mathrm{D}$ fast spin-echo T2-weighted sequence, echo time $(\mathrm{TE})=120 \mathrm{~ms}$ and repetition time (TR) $=3,900 \mathrm{~ms}$ was acquired for anatomical reference and IVD segmentation. To evaluate the biochemical composition of the IVDs, we used a T2 relaxometry multi-echo sequence as follows: $\mathrm{TE}=20 / 40 / 60 / 80 / 100 / 120 / 140 / 160 \mathrm{~ms}$ and TR $=3,000 \mathrm{~ms}$.

We generated T2 relaxometry maps using MINC tools and Display software (McConell Brain Imaging Centre, Montreal, Quebec, Canada) to extract the IVD relaxation time. T2 maps were computed on a pixel-by-pixel basis using an exponential decay model, whereas S0 is the equilibrium magnetisation signal and $\mathrm{S}(\mathrm{TE})$ is the signal acquired with the echo time (TE):

$$
S(T E)=S) * \exp \left(-\frac{T E}{T 2}\right)
$$

Using T2-weighted images, three circular ROIs placed on the NP, anterior annulus fibrosus (AAF), and posterior annulus fibrosus (PAF) by using 3 distinct sagittal sections and the complete volume of each lumbar IVD was manually segmented as previously described (Figure 3) (18). A second examiner performed IVD segmentations blindly and independently on all sample to assess inter-observer agreement. All segmentations were checked by the same senior radiologist and the values used for statistical analysis were the first examiner results.

\section{Statistical analysis}

The Shapiro-Wilk test was used to verify the data distribution. 

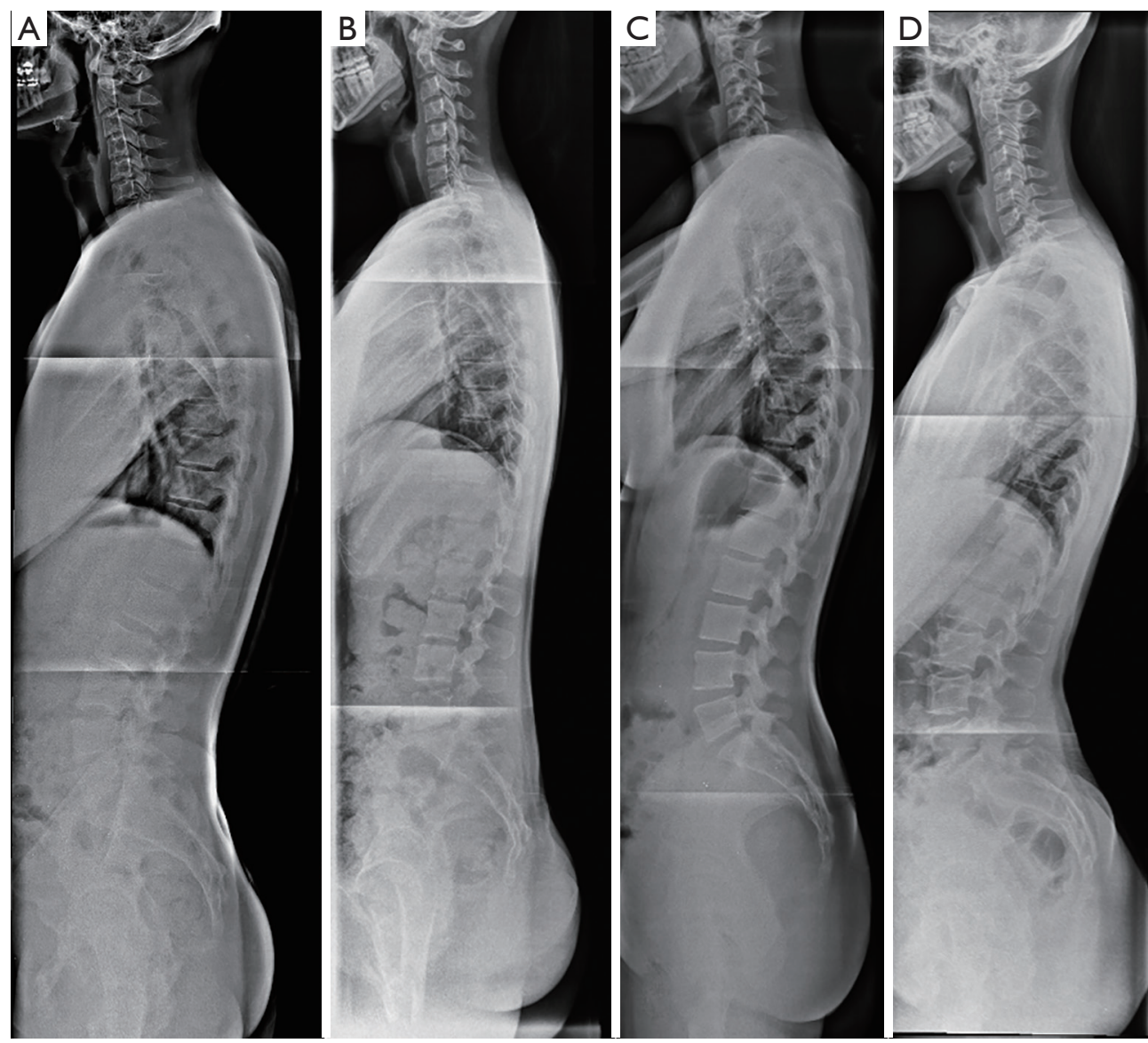

Figure 2 Panoramic radiographs of the spine presenting the four postural subtypes according to Roussouly's classification. (A) Roussouly Type 1; (B) Roussouly Type 2; (C) Roussouly Type 3; (D) Roussouly Type 4.
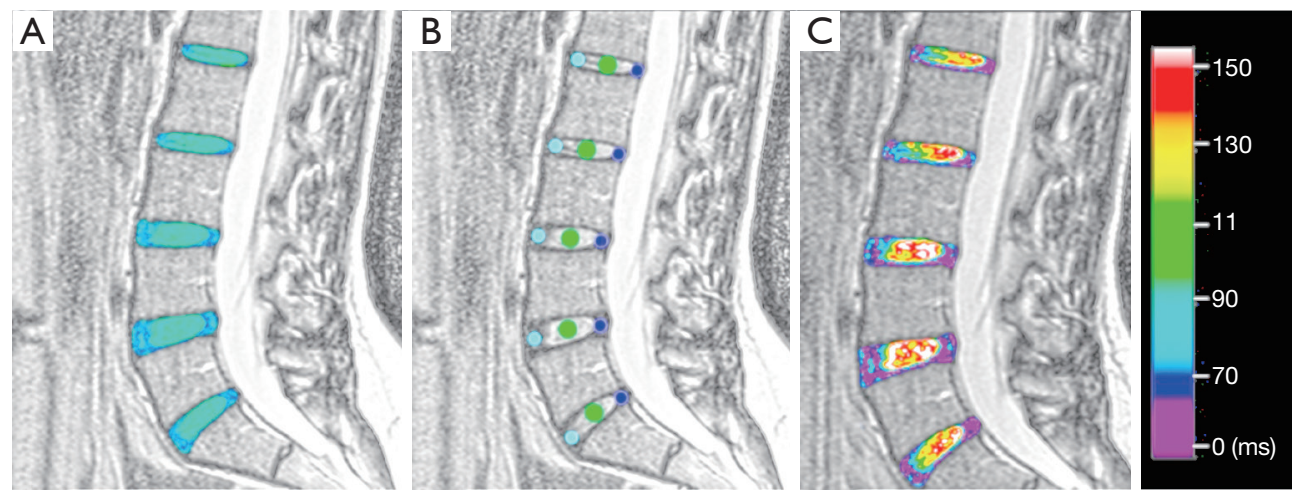

Figure 3 MRI analysing methods of lumbar intervertebral discs. (A) Manual segmentation of lumbar IVDs on the T2-weighted sagittal plane; (B) segmentation was performed on multiple contiguous sagittal slices encompassing the entire disc volume; (C) T2 relaxometry map of lumbar IVDs overlaid onto a T2-weighted image. 
Table 2 Intraclass correlation coefficients (ICC) and 95\% confidence intervals (CI) extracted from the IVD manual segmentation and spinopelvic parameter measurements

\begin{tabular}{lll}
\hline & ICC & $95 \% \mathrm{Cl}$ \\
\hline Disc segmentation & 0.92 & $0.85-0.95$ \\
SS & 0.93 & $0.88-0.96$ \\
PT & 0.77 & $0.59-0.87$ \\
PI & 0.91 & $0.85-0.96$ \\
LL & 0.93 & $0.87-0.96$ \\
TK & 0.88 & $0.85-0.97$ \\
TL & 0.90 & $0.88-0.96$ \\
CPA & 0.91 & $0.87-0.93$ \\
SVA & 0.89 & $0.81-0.91$ \\
SSA & 0.87 & $0.83-0.90$ \\
T1S1 & 0.93 & $0.90-0.96$ \\
L1S1 & 0.90 & $0.89-0.95$ \\
\hline
\end{tabular}

SS, sacral slope; PT, pelvic tilt; PI, pelvic incidence; LL, lumbar lordosis; TK, thoracic kyphosis; TL, thoracolumbar alignment; CPA, C2 pelvic angle; SVA, sagittal vertical axis; SSA, spinosacral angle.

The inter-observer agreement was assessed using the intraclass correlation coefficient (ICC) with a $95 \%$ confidence interval (95\% CI). Linear regression model with mixed effects (random and fixed effects) controlled by age was used to verify the spinopelvic influence on disc composition. The Pearson correlation were used to correlate the disc composition and volume with age, height, weight and Body Mass Index (BMI). To compare variables between the different groups, we used the unpaired t-test (between genders) and one-way ANOVA with a post-hoc Tukey to compare the Roussouly subtypes and T2 relaxometry values. Differences were considered statistically significant if $\mathrm{P}<0.05$. We used SAS v.9.4 (SAS Inst., Cary, NC) and GraphPad Prism v.5 (GraphPad Software, San Diego, California) for statistical analysis.

The statistical power (1- $\beta$ error probability) of our sample (93 subjects) was 0.839 with $\alpha$ error of $5 \%$.

\section{Results}

The mean age of the volunteers was $27.1 \pm 5.2$ years. The subjects showed a mean height of $1.71 \pm 0.08 \mathrm{~m}$, mean weight of $67.1 \pm 13.7 \mathrm{~kg}$, and mean body mass index (BMI) of $23.1 \pm 3.3 \mathrm{~kg} / \mathrm{m}^{2}$.

\section{Inter-observer agreement}

The ICC for T2 relaxometry values demonstrated substantial inter-observer agreement for manual disc segmentations at 0.92 (95\% CI: $0.85-0.95$ ). For spinopelvic parameters, the ICC was also substantial (ICC: $0.87-0.93$ ) (Table 2).

\section{IVD composition and spinopelvic balance parameters}

Table 3 shows the results as the means and standard deviations of the variables in our entire study group, including $\mathrm{T} 2$ relaxation time of the discs, disc volume and spinopelvic parameters. The mean disc relaxation time in men was: NP $=123.8 \pm 26.1 \mathrm{~ms}, \mathrm{PAF}=118.7 \pm 34.4 \mathrm{~ms}, \mathrm{AAF}=124.4 \pm 42.1 \mathrm{~ms}$; and in women: $\mathrm{NP}=122.8 \pm 26.4 \mathrm{~ms}, \mathrm{PAF}=121.8 \pm 33.2 \mathrm{~ms}$, $\mathrm{AAF}=122.1 \pm 45.8 \mathrm{~ms}$. There was not a significant difference between genders (NP: $\mathrm{P}=0.64$, $\mathrm{PAF}: \mathrm{P}=0.25, \mathrm{AAF}: \mathrm{P}=0.16$ ). However, the disc volume in men $\left(6.9 \pm 1.7 \mathrm{~cm}^{3}\right)$ and women $\left(5.6 \pm 1.6 \mathrm{~cm}^{3}\right)$ was significantly different $(\mathrm{P}=0.03)$.

Table 4 presents the correlations among anthropometric characteristics (age, height, weight and BMI), IVD T2 relaxation times and volumes. Age was inversely correlated with both $\mathrm{T} 2$ relaxation times and volumes for all segments. The IVD volume showed a moderate correlation with the height of the volunteers. The L3L4 disc volume showed a weak correlation with age, height, weight and BMI.

Regarding the spinopelvic parameters, most variables did not differ between men and women, with the exception of TK $\left(\right.$ men $=40.7^{\circ} \pm 11.3^{\circ}$ and women $=34.3^{\circ} \pm 10.2^{\circ}(\mathrm{P}=0.004)$, SVA $($ men $=15.3 \pm 17.9 \mathrm{~mm}$ and women $=0.6 \pm 19.7 \mathrm{~mm}$ $(\mathrm{P}=0.003)$ and T1S1 length $(\mathrm{men}=52.4 \pm 2.7 \mathrm{~cm}$ and women $=48.6 \pm 2.7 \mathrm{~cm}(\mathrm{P}<0.0001)$.

\section{Relationships among spinopelvic parameters and IVD composition}

Table 5 presents the vertebral curves effects on disc composition. The increase in lumbar lordosis influenced the increase of $\mathrm{T} 2$ relaxation times in all nucleus pulposus: L1L2, L2L3, L3L4, L4L5 and L5S1; PAF: L2L3, L3L4, L4L5 and L5S1; and anterior annulus fibrosus: L1L2, L2L3, L3L4, L4L5. The increase in thoracic kyphosis reflected on increase in NP: L3L4, PAF: L2L3, L3L4, L4L5 and L5S1, AAF: L1L2 and L3L4. The thoracolumbar alignment increase influenced the decrease on NP L1L2 and increase on L3L4.

Table 6 shows the parameters pelvic incidence, pelvic tilt and sacral slope effects on disc composition. The increase 
Table 3 Lumbar disc T2 relaxation times, disc volumes and spinopelvic parameters of asymptomatic young adults expressed as the mean and standard deviation (SD)

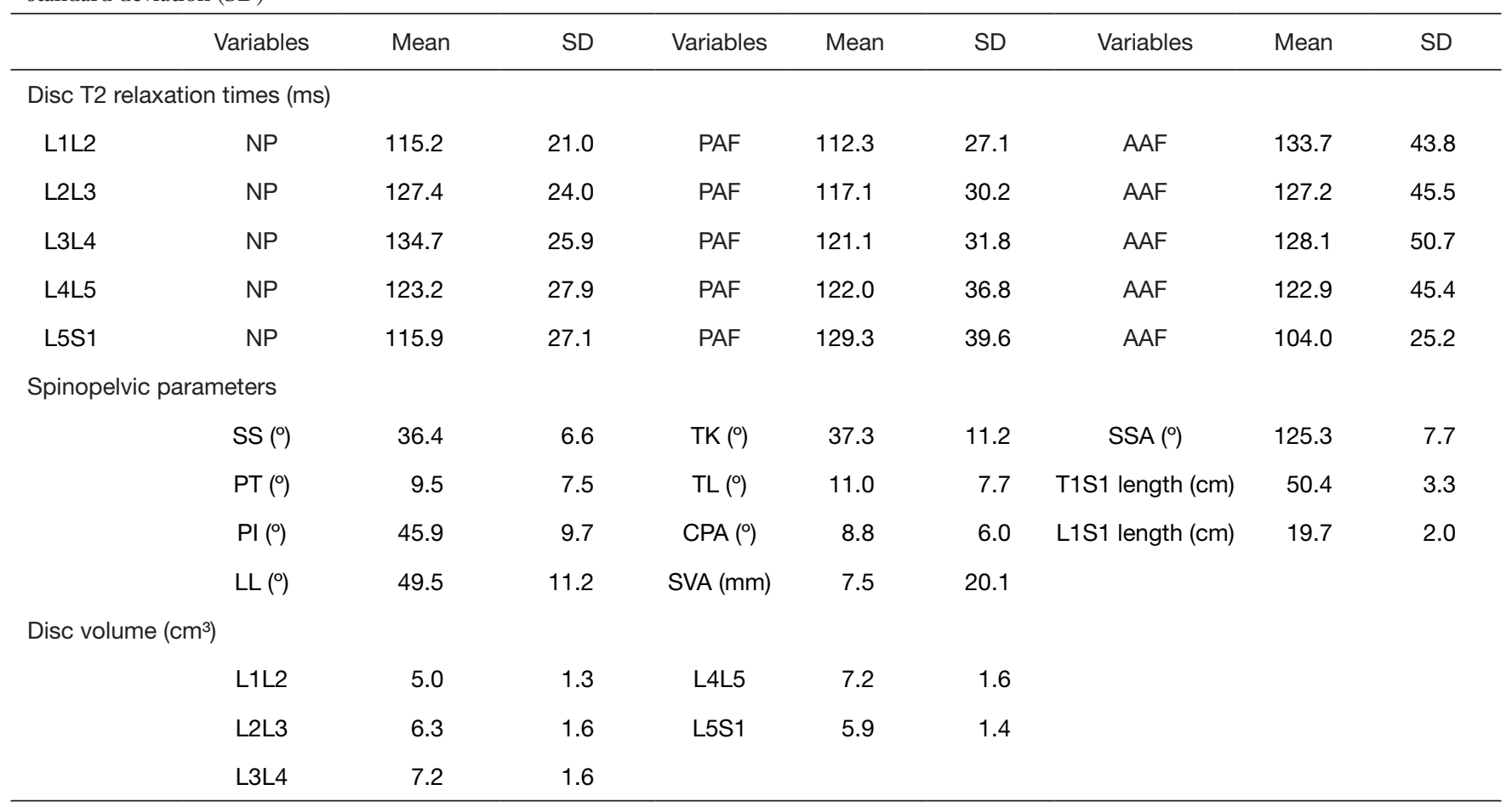

NP, nucleus pulposus; PAF, posterior annulus fibrosus; AFA, anterior annulus fibrosus; SS, sacral slope; PT, pelvic tilt; PI, pelvic incidence; LL, lumbar lordosis; TK, thoracic kyphosis; TL, thoracolumbar alignment; CPA, C2 pelvic angle; SVA, sagittal vertical axis; SSA, spinosacral angle.

Table 4 Correlation values $(\mathrm{R})$ between the anthropometric characteristics with the $\mathrm{T} 2$ relaxation times and disc volumes at each lumbar level

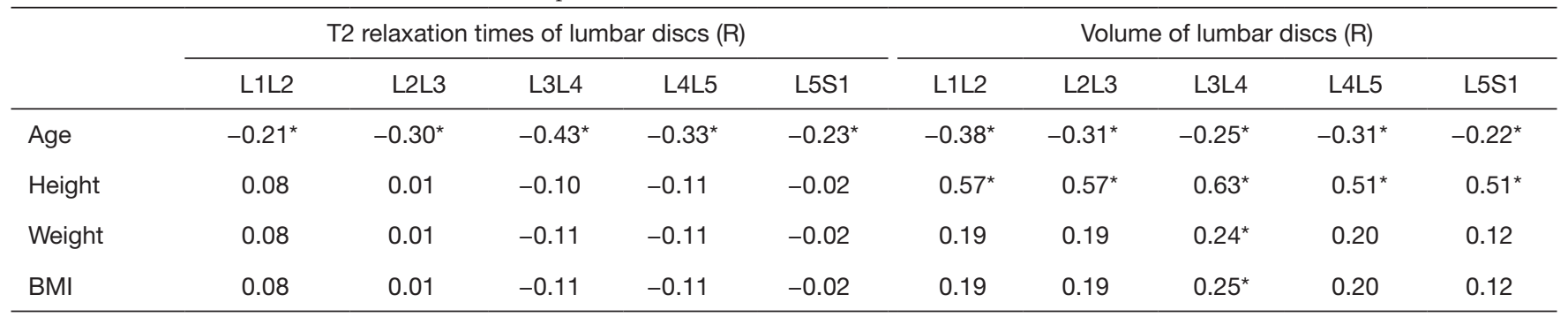

*indicates a significant correlations $(\mathrm{P}<0.05)$.

on Pelvic Tilt influenced the increase in NP relaxation time: L2L3 and L3L4, and a decrease on AAF: L5S1. The increase in Pelvic Incidence influenced the increase in NP: L2L3, L3L4 and L4L5. The increase in Sacral Slope influenced the increase in NP: L3L4, PAF: L2L3, L3L4, L4L5 and L5S1; AAF: L1L2, L2L3, L3L4 and L4L5.

Table 7 presented SVA, CPA, SSA, L1S1 and T1S1 length possible effects on disc composition. High values of SVA implicates on reduction in L5S1 NP relaxation time. SSA influence anterior and posterior annulus fibrosus in all lumbar spine. CPA values presented an association with L3L4 nucleus pulposus. The L1S1 length performs an influence on nucleus pulposus relaxation times of L2L3, L3L4, L4L5 and L5S1

\section{Disc composition analysis according to the Roussouly classification}

The volunteer distribution according Roussouly classification was: Type $1, \mathrm{n}=21$; Type 2, n=20; Type 3, $\mathrm{n}=28$; and Type 4, n=24. 
Table 5 Effects of spinal curves on disc composition measured by linear regression with mixed effects controlled by age

\begin{tabular}{|c|c|c|c|c|c|c|c|c|c|c|c|c|}
\hline \multirow[b]{2}{*}{ LL } & \multicolumn{4}{|c|}{ Nucleus pulposus (NP) } & \multicolumn{4}{|c|}{ Posterior annulus fibrosus (PAF) } & \multicolumn{4}{|c|}{ Anterior annulus fibrosus (AAF) } \\
\hline & Effect $(\beta)$ & $\mathrm{P}$ & & & Effect $(\beta)$ & $\mathrm{P}$ & & & Effect $(\beta)$ & $\mathrm{P}$ & & \\
\hline L1L2 & 0.42 & $<0.01^{*}$ & 0.14 & 0.71 & 0.43 & 0.05 & -0.01 & 0.86 & 0.96 & $0.00^{\star}$ & 0.38 & 1.53 \\
\hline L2L3 & 0.67 & $<0.01^{*}$ & 0.38 & 0.96 & 0.51 & $0.02^{*}$ & 0.08 & 0.94 & 0.87 & $0.00^{*}$ & 0.29 & 1.44 \\
\hline L4L5 & 0.59 & $<0.01^{*}$ & 0.30 & 0.87 & 0.65 & $0.00^{*}$ & 0.22 & 1.08 & 0.75 & $0.01^{*}$ & 0.17 & 1.33 \\
\hline L5S1 & 0.45 & $<0.01^{*}$ & 0.17 & 0.74 & 0.78 & $0.00^{*}$ & 0.35 & 1.22 & 0.36 & 0.22 & -0.22 & 0.94 \\
\hline Mean & 0.59 & $<0.01^{*}$ & 0.31 & 0.86 & 0.60 & $0.01^{*}$ & 0.17 & 1.02 & 0.76 & $0.01^{*}$ & 0.19 & 1.33 \\
\hline \multicolumn{13}{|l|}{ TK } \\
\hline L3L4 & 0.43 & $<0.01^{*}$ & 0.11 & 0.74 & 0.60 & $0.01^{*}$ & 0.16 & 1.05 & 0.62 & $0.04^{\star}$ & 0.02 & 1.22 \\
\hline L4L5 & 0.19 & 0.23 & -0.12 & 0.51 & 0.65 & $0.00^{*}$ & 0.20 & 1.09 & 0.51 & 0.10 & -0.09 & 1.11 \\
\hline L5S1 & 0.01 & 0.94 & -0.30 & 0.33 & 0.81 & $0.00^{*}$ & 0.37 & 1.25 & 0.02 & 0.95 & -0.59 & 0.62 \\
\hline Mean & 0.17 & 0.26 & -0.13 & 0.48 & 0.58 & $0.01^{*}$ & 0.15 & 1.01 & 0.49 & 0.10 & -0.09 & 1.07 \\
\hline \multicolumn{13}{|l|}{$\mathrm{TL}$} \\
\hline L1L2 & -0.67 & $0.01^{*}$ & -1.18 & -0.15 & -0.29 & 0.43 & -1.02 & 0.43 & 0.80 & 0.10 & -0.16 & 1.76 \\
\hline L2L3 & 0.14 & 0.58 & -0.37 & 0.66 & -0.03 & 0.93 & -0.76 & 0.69 & 0.36 & 0.47 & -0.61 & 1.32 \\
\hline L3L4 & 0.56 & 0.04 & 0.04 & 1.07 & 0.20 & 0.59 & -0.52 & 0.92 & 0.49 & 0.31 & -0.47 & 1.46 \\
\hline
\end{tabular}

LL, lumbar lordosis; TK, thoracic kyphosis; TL, thoracolumbar alignment. * $\mathrm{P}<0.05$.

There were no significant differences between the Roussouly subtypes in terms of $\mathrm{T} 2$ relaxation or volume at any nucleus pulposus. There is statistical significant difference on posterior annulus fibrosus of L4L5 disc $(\mathrm{P}=0.01)$, where Type $1(109.1 \pm 33.2 \mathrm{~ms})$ was significantly lower than Type $4(136.6 \pm 33.8 \mathrm{~ms})$. Also, we found a significant statistical difference on posterior annulus fibrosus of L3L4 disc, the Type $2(108.3 \pm 20.3 \mathrm{~ms})$ was significantly lower than Type $4(134.9 \pm 35.3 \mathrm{~ms})(\mathrm{P}=0.03)$.

\section{Discussion}

Our results showed that a decrease in lumbar lordosis associates with discrete disc dehydration at all lumbar spine levels. There is a linear relationship between lordosis magnitude and nucleus pulposus and annulus fibrosus relaxometry at all lumbar intervertebral discs. Pelvic incidence and pelvic tilt decrease were accompanied by NP relaxation time reduction, and sacral slope decrease associates with reduced annulus fibrosus $\mathrm{T} 2$ relaxation. Thoracic kyphosis and SSA decrease correlates with dehydration on annulus fibrosus.

Three studies have demonstrated the relationship between spinopelvic balance and IVD degeneration using the semiquantitative Pfirrmann classification (19-21). However, we did not identify studies that assessed the potential correlation between spinal sagittal balance parameters and quantitative MRI techniques. The T2 relaxation is considered a biological marker of ageing or early degenerative disc processes $(15,22)$.

Subjects with clinically significant IVD disease have a 
Table 6 Effects of pelvic tilt, pelvic incidence and sacral slope on disc composition measured by linear regression with mixed effects controlled by age

\begin{tabular}{|c|c|c|c|c|c|c|c|c|c|c|c|c|}
\hline & \multicolumn{4}{|c|}{ Nucleus pulposus (NP) } & \multicolumn{4}{|c|}{ Posterior annulus fibrosus (PAF) } & \multicolumn{4}{|c|}{ Anterior annulus fibrosus (AAF) } \\
\hline \multicolumn{13}{|l|}{$\mathrm{PT}$} \\
\hline L1L2 & 0.09 & 0.74 & -0.45 & 0.64 & -0.46 & 0.24 & -1.23 & 0.31 & 0.92 & 0.08 & -0.10 & 1.94 \\
\hline L2L3 & 0.76 & $<0.01^{*}$ & 0.22 & 1.30 & -0.22 & 0.58 & -0.99 & 0.55 & 0.57 & 0.28 & -0.45 & 1.58 \\
\hline L4L5 & 0.30 & 0.28 & -0.25 & 0.84 & 0.02 & 0.96 & -0.75 & 0.79 & 0.20 & 0.70 & -0.82 & 1.22 \\
\hline L5S1 & -0.26 & 0.34 & -0.81 & 0.28 & 0.49 & 0.21 & -0.28 & 1.26 & -1.25 & $0.02^{*}$ & -2.27 & -0.23 \\
\hline Mean & 0.42 & 0.07 & -0.03 & 0.88 & -0.02 & 0.94 & -0.69 & 0.64 & 0.19 & 0.68 & -0.70 & 1.08 \\
\hline \multicolumn{13}{|l|}{$\mathrm{PI}$} \\
\hline L3L4 & 0.62 & $<0.01^{*}$ & 0.27 & 0.97 & 0.31 & 0.24 & -0.20 & 0.83 & 0.48 & 0.17 & -0.20 & 1.17 \\
\hline L4L5 & 0.38 & $0.03^{*}$ & 0.03 & 0.73 & 0.34 & 0.19 & -0.17 & 0.86 & 0.34 & 0.33 & -0.35 & 1.03 \\
\hline L5S1 & 0.21 & 0.23 & -0.14 & 0.56 & 0.50 & 0.06 & -0.02 & 1.01 & -0.07 & 0.84 & -0.76 & 0.62 \\
\hline Mean & 0.38 & $0.03^{*}$ & 0.04 & 0.72 & 0.30 & 0.24 & -0.20 & 0.81 & 0.36 & 0.29 & -0.31 & 1.04 \\
\hline \multicolumn{13}{|l|}{ SS } \\
\hline L1L2 & 0.14 & 0.61 & -0.38 & 0.66 & 0.74 & 0.05 & 0.00 & 1.47 & 1.19 & $0.02^{*}$ & 0.19 & 2.18 \\
\hline L2L3 & 0.46 & 0.08 & -0.06 & 0.98 & 0.86 & 0.02 & 0.13 & 1.60 & 1.05 & $0.04^{\star}$ & 0.05 & 2.04 \\
\hline L3L4 & 0.66 & $0.01^{*}$ & 0.14 & 1.18 & 0.97 & 0.01 & 0.24 & 1.71 & 1.06 & $0.04^{*}$ & 0.07 & 2.06 \\
\hline
\end{tabular}

PT, pelvic tilt; PI, pelvic incidence; SS, sacral slope. ${ }^{*} \mathrm{P}<0.05$.

tendency to present with LL rectification, low SS and PI, and a progressive compensatory posterior pelvic tilt (23-25). In horizontal vertebral endplates, the axial load acts predominantly on the discs, and contributes to IVD wear (26). We encountered a slight trend towards greater disc dehydration at all disc levels at the lower magnitudes of segmental lordosis. To explain the probable biomechanical influence on the degeneration mechanism, Huang et al. (27) showed that IVDs exposed to an increased axial load secondary to LL rectification exhibited reduced expression of aggrecan molecules, reduced collagen type I and II expression, and increased ADAMT synthesis, which indicated metalloproteinase degradation. The loss of proteoglycans is associated with the loss of the water content (1), which is detectable as a decrease in the T2 relaxometry. A systematic meta-analysis performed by Chun et al. (28) concluded that low back pain with disc degeneration or herniation is associated with loss of LL. It was interesting to note that in cases of nonspecific low back pain, this LL difference between symptomatic and the healthy control group was lower (20). Our results showed that lumbar lordosis reduction is associated with NP water content decrease even in subjects without pain, pointing in the same direction. The PAF, especially at L3L4, L4L5 and L5S1, accompanying the degenerative process in NP and suffer from these overloads.

TK and thoracolumbar curvatures correlated with T2 relaxometry in the annulus fibrosus. Generally, the shape of the TK follows the changes in LL (29). In our study, we observed that the kyphotic angle corroborated lordosis 
Table 7 Effects of global spinopelvic parameters on disc composition measured by linear regression with mixed effects controlled by age

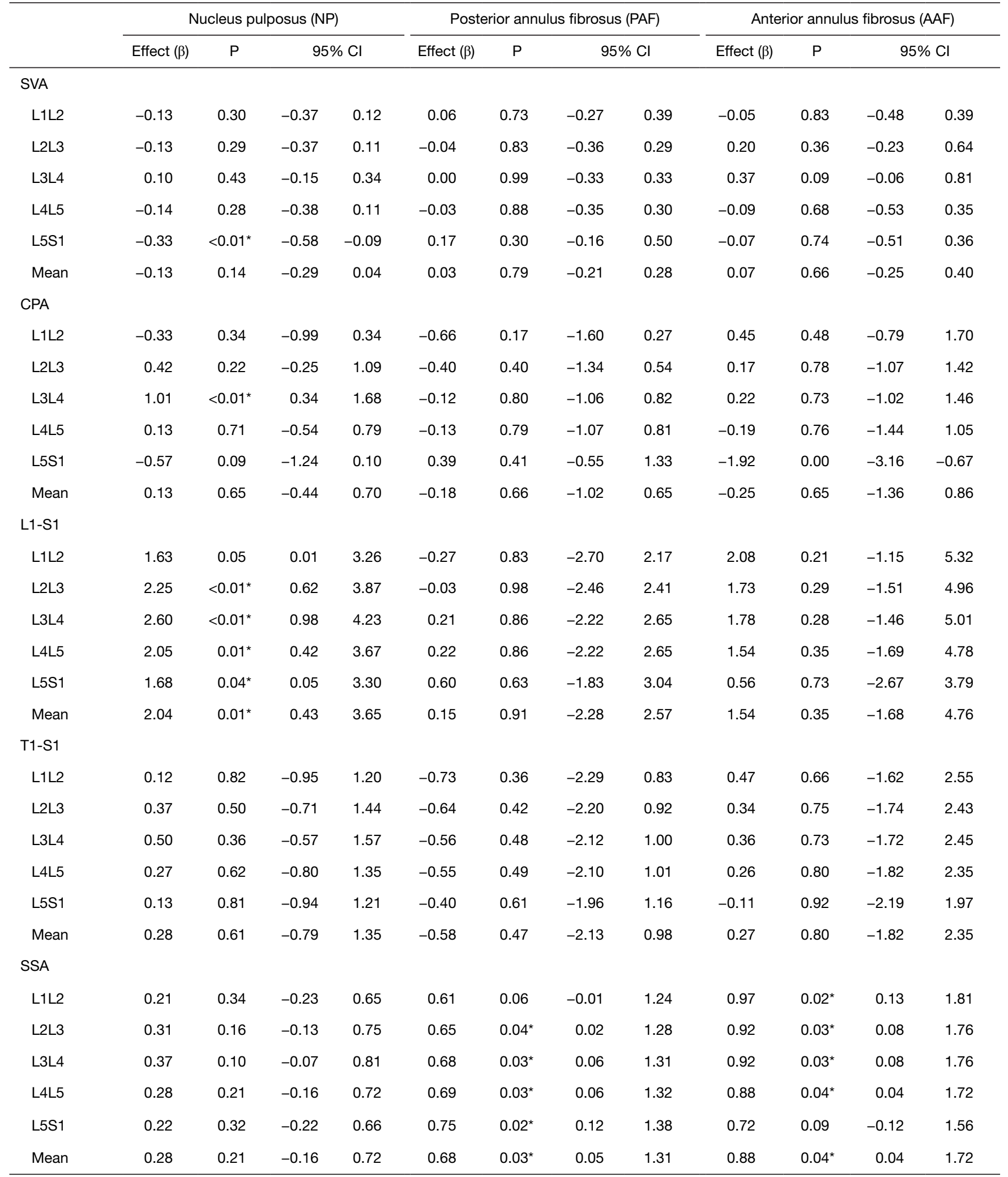

SVA, sagittal vertical axis; CPA, C2 pelvic angle; L1-S1, L1 to S1 length; T1-S1, T1 to S1 length; SSA, spinosacral angle. ${ }^{*} \mathrm{P}<0.05$. 
on the hydration status on PAF. The kyphotic angle and the thoracolumbar alignment affect specially L1L2 and L3L4 discs. Based on the analysis of the spine as a whole, the curve transition disc and central disc are the most overloaded.

SS also have a weak influence on disc composition and this was most evident in the annulus fibrosus and L3L4 nucleus pulposus. Oh and Eun (23) found no correlation between LL and degeneration in young adults but suggested that SS could be an associated factor. These angle decrease seems to cause a greater overload mainly in the posterior portion of the annulus, which could lead to the lamellae fragility and contribute to an acceleration of nuclear degeneration (1). The pelvis is the cornerstone of spinal sagittal alignment and several measurements have been developed to relate the morphology of the pelvis to sagittal spinal alignment (30). It was interesting to note that PI and PT influenced the values L2L3 to L4L5 discs. The pelvic parameters changes are associated with disc degeneration on lumbar spine as a whole, but we not found studies that did a discriminated level-for-level analysis for comparison purposes. Ghasemi et al. (31) reported that the sacral angles were not an isolated factor for degeneration, perhaps this result can be derived from a factors conjunction and not an isolated fact.

SSA, SVA and CPA are used to evaluate spinal imbalance. In our volunteers, lower SSA values are associated with decreased T2 values in the posterior annulus fibrosus from $\mathrm{L} 2 \mathrm{~L} 3$ to $\mathrm{L} 5 \mathrm{~S} 1$, as well as in the anterior annulus fibrosus of L1L2 to L4L5. However, again this association is low as can be seen in the analysis of confidence intervals. The SSA is also decreased in disc related diseases such as degenerative kyphosis (32), rheumatoid arthritis, functional abnormalities (33), discogenic low back pain (34). SVA above $5 \mathrm{~cm}$ is considered a criterion of sagittal imbalance and may be associated with spondylolisthesis (35) and is also moderately correlated with clinically relevant dysfunctions (ODI >40) (36). Our sample consisted of asymptomatic subjects and such parameters were always within normal values for all volunteers $(37,38)$. It is interesting to note that SVA values vary widely between Roussouly subtypes even in asymptomatic people (39). Therefore, our results may only allow concluding that global spinopelvic parameters do not associate with IVD volume and composition, in individuals without sagittal plane imbalance. Future studies including symptomatic and imbalanced patients are necessary for better investigation.

When loads applied to the IVD are physiological, a restoration capability of the proteoglycan content is present even in degenerated discs (40). Because our sample consisted of asymptomatic volunteers and the spine posture in these subjects could be considered within normal limits, we presume that our findings reflect physiological mechanical loads on the lumbar discs. Therefore, our results suggest that the spinopelvic balance may not exert a strong influence on IVD ageing or the early disc degenerative process. Presumably, other risk factors are more important. For instance, age has been described as a major contributor to the progression of degeneration (14). In our sample of young asymptomatic adults, we found a tendency for a decreased relaxation time in all lumbar IVDs in the age range between 20 and 40 years, which was consistent with the literature $(13,41)$. It was interesting to note that in our asymptomatic sample, the spinopelvic parameters presented more relationships with the annulus fibrosus than the nucleus pulposus. Perhaps these biomechanical factors can further alter the laminar structure of the annulus, making it more fragile and in order to influence a future loss of nuclear water content.

However, the effect of gender on disc degeneration is controversial. We did not find significant differences in IVD T2 relaxation times between genders, although male subjects showed greater IVD volumes. Sieminow et al. (42) and Pfirrmann et al. (43) found no significant differences in disc degeneration between men and women, whereas other studies have described a gender influence $(44,45)$.

We detected correlations between volunteer height and IVD volume. Furthermore, a moderate correlation was detected between the T1S1 length and the IVD volume, and a weak correlation between L1S1 length and L4L5 NP T2 values. The IVDs accommodate various biomechanical forces (1). The discs can bear physiological loadings represented by dynamic compressions at $1 \mathrm{MPa}$ and $0.2 \mathrm{~Hz}$ while still preserving cell metabolism and viability (46). However, for most IVD levels, weight and BMI showed no influence on the IVD composition in our study. Zobel et al. (45) found that the disc composition correlated with the BMI in subjects who were overweight, but the volunteers in our study group were not overweight.

Previous studies have confirmed that genetics are a strong risk factor for disc degeneration. Battié and Videman (47) found a substantial genetic influence on lumbar disc degeneration. The risk of herniation or degeneration in individuals younger than 21 years old is four to five times higher in patients with a positive family history. Biological processes associated with the synthesis and degradation of 
biochemical components may be genetically predetermined, causing accelerated degenerative changes. Stone et al. (48) reported a strong genetic influence on spine curvatures and described an inverse association between LL and the lumbar disc degeneration prevalence. These authors also found that subjects with disc degeneration showed a nearly 3.2 higher risk of developing low back pain in the future. Mechanical stress caused by intense asymmetrical repetitive movements is a stronger trigger for IVD degeneration (49). Genetic effects on the spinal structures shape can affect mechanical properties and therefore the vulnerability of the spine to such external forces (48). Physical activity level has also been identified as a protective factor for disc degeneration (6). We chose to include only non-athlete subjects to avoid this possible confounding factor in the analysis of IVD composition.

Patients of different Roussouly subtypes showed differences on posterior annulus fibrosus for: Type 1 versus Type 4 at L4L 5 level, and Type 2 versus Type 4 at L3L4 level. A previous study identified correlation between the degree of disc degeneration according to Pfirrmann classification and LL only at the L4L5 level (20). Patients with Roussouly subtype 2 exhibited a higher prevalence of degenerated discs than those with subtype 4 (20). On the other hand, Torrie et al. (19) found no differences in lumbar IVD degeneration frequency between patients of different Roussouly subtypes. It is possible to perceive that the differences appear between very different subtypes (Type 1 and 2 have low lordosis while Type 4 exhibits high values of lumbar lordosis), however these differences are punctual. Maybe stratify the sample into subtypes, do not show the true effects, since the variables are continuous and not categorical.

Our study has limitations that deserve mention. First, we performed a cross-sectional evaluation and could not evaluate cause and effect relationships between disc degeneration and spinopelvic parameters. The ideal would be to have a follow-up of the volunteers for at least 3 to 5 years. A longitudinal study is desirable to assess cause and effect relationship. In addition, our sample was composed exclusively of asymptomatic and non-athletic individuals and therefore, our results may not be applicable to symptomatic patients. Our initial hypothesis was that changes in spinopelvic parameters would be associated with mechanical load differences and consequently affecting the intervertebral disc composition. We found that this correlation exists but it is less than we supposed. We chose to evaluate young, asymptomatic individuals because our interest in this project was to detect early disc changes potentially related to physiological postural variations and spinopelvic parameters. Older symptomatic individuals often have multiple segments with discopathy and advanced disc space collapse, which could hinder relaxometry measurements. Perhaps our results contribute to reduce the tendency to overestimate biomechanics as a risk factor for disc degeneration. Future studies are necessary to investigate symptomatic patients with spine degenerative disease to confirm probable correlation between spinopelvic parameters and spine imbalance and IVD composition.

\section{Conclusions}

Lower magnitudes of lumbar lordosis, thoracic kyphosis, thoracolumbar alignment, pelvic tilt, pelvic incidence, sacral slope and spino sacral angle are associated with intervertebral disc dehydration. Contrary to our initial hypothesis, our results favours that biomechanical factors may not have a strong contribution to the initial degeneration of the lumbar IVD in young adults.

\section{Acknowledgments}

We thank the Science Center of Images and Medical Physics of Ribeirão Preto Medical School for their support. Funding: This study was funded by the National Council of Technological and Scientific Development (CNPq, Grant no. 473688/2012-3), the Coordination for the Improvement of Higher Level Personnel (CAPES, Scholarship Grant no. 01500990612, Finance Code 001), and the Financing of Studies and Projects (FINEP, 01/2006 Ref. 0184/07) of Brazil.

\section{Footnote}

Conflicts of Interest: All authors have completed the ICMJE uniform disclosure form (available at http://dx.doi. org/10.21037/qims-20-40). Dr. MHNB serves as an unpaid editorial board member of Quantitative Imaging in Medicine and Surgery. The other authors have no conflicts of interest to declare.

Ethical Statement: The study was approved by institutional committee ethics board of Ribeirão Preto Medical School, University of São Paulo (No. 4236/2012) and informed consent was taken from all the patients. Written informed consent was obtained from the patient for publication of this study and any accompanying images. A copy of the 
written consent is available for review by the Editor-inChief of this journal.

Open Access Statement: This is an Open Access article distributed in accordance with the Creative Commons Attribution-NonCommercial-NoDerivs 4.0 International License (CC BY-NC-ND 4.0), which permits the noncommercial replication and distribution of the article with the strict proviso that no changes or edits are made and the original work is properly cited (including links to both the formal publication through the relevant DOI and the license). See: https://creativecommons.org/licenses/by-nc-nd/4.0/.

\section{References}

1. Wang F, Cai F, Shi R, Wang XH, Wu XT. Aging and age related stresses: a senescence mechanism of intervertebral disc degeneration. Osteoarthritis Cartilage 2016;24:398-408.

2. Risbud MV, Shapiro IM. Role of cytokines in intervertebral disc degeneration: pain and disc content. Nat Rev Rheumatol 2014;10:44-56.

3. Wu X, Zhuang S, Mao Z, Chen H. Microendoscopic discectomy for lumbar disc herniation: surgical technique and outcome in 873 consecutive cases. Spine (Phila Pa 1976) 2006;31:2689-94.

4. Brinjikji W, Diehn FE, Jarvik JG, Carr CM, Kallmes DF, Murad MH, Luetmer PH. MRI Findings of Disc Degeneration are More Prevalent in Adults with Low Back Pain than in Asymptomatic Controls: A Systematic Review and Meta-Analysis. AJNR Am J Neuroradiol 2015;36:2394-9.

5. Takatalo J, Karppinen J, Taimela S, Niinimäki J, Laitinen J, Blanco Sequeiros R, Paananen M, Remes J, Näyhä S, Tammelin T, Korpelainen R, Tervonen O. Body mass index is associated with lumbar disc degeneration in young Finnish males: subsample of Northern Finland birth cohort study 1986. BMC Musculoskelet Disord 2013;14:87.

6. Elfering A, Semmer N, Birkhofer D, Zanetti M, Hodler J, Boos N. Risk factors for lumbar disc degeneration: a 5 -year prospective MRI study in asymptomatic individuals. Spine (Phila Pa 1976) 2002;27:125-34.

7. Tsouknidas A, Sarigiannidis SO, Anagnostidis K, Michailidis N, Ahuja S. Assessment of stress patterns on a spinal motion segment in healthy versus osteoporotic bony models with or without disc degeneration: a finite element analysis. Spine J 2015;15:S17-22.
8. Lamartina C, Berjano P, Petruzzi M, Sinigaglia A, Casero G, Cecchinato R, Damilano M, Bassani R. Criteria to restore the sagittal balance in deformity and degenerative spondylolisthesis. Eur Spine J 2012;21 Suppl 1:S27-31.

9. Lafage V, Schwab F, Skalli W, Hawkinson N, Gagey PM, Ondra S, Farcy JP. Standing balance and sagittal plane spinal deformity: analysis of spinopelvic and gravity line parameters. Spine (Phila Pa 1976) 2008;33:1572-8.

10. Barrey C, Roussouly P, Le Huec JC, D'Acunzi G, Perrin G. Compensatory mechanisms contributing to keep the sagittal balance of the spine. Eur Spine J 2013;22 Suppl 6:S834-41.

11. Vergroesen PP, Kingma I, Emanuel KS, Hoogendoorn RJ, Welting TJ, van Royen BJ, van Dieën JH, Smit TH. Mechanics and biology in intervertebral disc degeneration: a vicious circle. Osteoarthritis Cartilage 2015;23:1057-70.

12. Lotz JC, Haughton V, Boden SD, An HS, Kang JD, Masuda K, Freemont A, Berven S, Sengupta DK, Tanenbaum L, Maurer P, Ranganathan A, Alavi A, Marinelli NL. New treatments and imaging strategies in degenerative disease of the intervertebral disks. Radiology 2012;264:6-19.

13. Marinelli NL, Haughton VM, Anderson PA. T2 relaxation times correlated with stage of lumbar intervertebral disk degeneration and patient age. AJNR Am J Neuroradiol 2010;31:1278-82.

14. Antoniou J, Pike GB, Steffen T, Baramki H, Poole AR, Aebi M, Alini M. Quantitative magnetic resonance imaging in the assessment of degenerative disc disease. Magn Reson Med 1998;40:900-7.

15. Mwale F, Iatridis JC, Antoniou J. Quantitative MRI as a diagnostic tool of intervertebral disc matrix composition and integrity. Eur Spine J 2008;17 Suppl 4:432-40.

16. Marks M, Stanford C, Newton P. Which lateral radiographic positioning technique provides the most reliable and functional representation of a patient's sagittal balance? Spine (Phila Pa 1976) 2009;34:949-54.

17. Roussouly P, Gollogly S, Berthonnaud E, Dimnet J. Classification of the normal variation in the sagittal alignment of the human lumbar spine and pelvis in the standing position. Spine (Phila Pa 1976) 2005;30:346-53.

18. Menezes-Reis R, Salmon CE, Carvalho CS, Bonugli GP, Chung CB, Nogueira-Barbosa MH. T1 $\rho$ and T2 mapping of the intervertebral disk: comparison of different methods of segmentation. AJNR Am J Neuroradiol 2015;36:606-11.

19. Torrie PAG, McKay G, Byrne R, Morris SAC, Harding IJ. The influence of lumbar spinal subtype on lumbar Intervertebral disc degeneration in young and middle-aged 
adults. Spine Deformity 2015;3:172-9.

20. Menezes-Reis R, Bonugli GP, Dalto VF, Herrero CF, Defino HL, Nogueira-Barbosa MH. The Association Between Lumbar Spine Sagittal Alignment And L4-L5 Disc Degeneration Among Asymptomatic Young Adults. Spine (Phila Pa 1976) 2016;41:E1081-7.

21. Mardare M, Oprea M, Popa I, Zazgyva A, Niculescu M, Poenaru DV. Sagittal balance parameters correlate with spinal conformational type and MRI changes in lumbar degenerative disc disease: results of a retrospective study. Eur J Orthop Surg Traumatol 2016;26:735-43.

22. Menezes-Reis R, Salmon CEG, Bonugli GP, Mazoroski

D, Tamashiro MH, Savarese LG, Nogueira-Barbosa MH. Lumbar intervertebral discs T2 relaxometry and T1 $\rho$ relaxometry correlation with age in asymptomatic young adults. Quant Imaging Med Surg 2016;6:402-12.

23. Oh YM, Eun JP. Clinical Impact of Sagittal Spinopelvic Parameters on Disc Degeneration in Young Adults. Medicine (Baltimore) 2015;94:e1833.

24. Rajnics P, Templier A, Skalli W, Lavaste F, Illes T. The importance of spinopelvic parameters in patients with lumbar disc lesions. Int Orthop 2002;26:104-8.

25. Endo K, Suzuki H, Tanaka H, Kang Y, Yamamoto K. Sagittal spinal alignment in patients with lumbar disc herniation. Eur Spine J 2010;19:435-8.

26. Roussouly P, Pinheiro-Franco JL. Biomechanical analysis of the spino-pelvic organization and adaptation in pathology. Eur Spine J 2011;20 Suppl 5:609-18.

27. Huang M, Wang HQ, Zhang Q, Yan XD, Hao M, Luo ZJ. Alterations of ADAMTSs and TIMP-3 in human nucleus pulposus cells subjected to compressive load: Implications in the pathogenesis of human intervertebral disc degeneration. J Orthop Res 2012;30:267-73.

28. Chun SW, Lim CY, Kim K, Hwang J, Chung SG. The relationships between low back pain and lumbar lordosis: a systematic review and meta-analysis. Spine J 2017;17:1180-91.

29. Keller TS, Colloca CJ, Harrison DE, Harrison DD, Janik TJ. Influence of spine morphology on intervertebral disc loads and stresses in asymptomatic adults: implications for the ideal spine. Spine J 2005;5(3):297-309.

30. Ruiz Santiago F, Ramos-Bossini AJL, Wang YXJ, Zúñiga DL. The role of radiography in the study of spinal disorders. Quant Imaging Med Surg 2020;10:2322-55.

31. Ghasemi A, Haddadi K, Khoshakhlagh M, Ganjeh HR. The Relation Between Sacral Angle and Vertical Angle of Sacral Curvature and Lumbar Disc Degeneration: A CaseControl Study. Medicine (Baltimore) 2016;95:e2746.
32. Son SM, Shin JK, Goh TS, Suh KT, Lee JS. Predictive Findings of the Presence of Stooping in Patients With Lumbar Degenerative Kyphosis by Upright Whole Spine Lateral Radiography. Spine (Phila Pa 1976) 2018;43:571-7.

33. Lee HS, Shin JK, Goh TS. Correlations Between Sagittal Spinal Balance and Quality of Life in Rheumatoid Arthritis. Clin Spine Surg 2017;30:E412-7.

34. Pellet N, Aunoble S, Meyrat R, Rigal J, Le Huec JC. Sagittal balance parameters influence indications for lumbar disc arthroplasty or ALIF. Eur Spine J 2011;20 Suppl 5:647-62.

35. Gille O, Challier V, Parent H, Cavagna R, Poignard A, Faline A, Fuentes S, Ricart O, Ferrero E, Ould Slimane M; French Society of Spine Surgery (SFCR). Degenerative lumbar spondylolisthesis: cohort of 670 patients, and proposal of a new classification. Orthop Traumatol Surg Res 2014;100:S311-5.

36. Ailon T, Smith JS, Shaffrey CI, Lenke LG, Brodke D, Harrop JS, Fehlings M, Ames CP. Degenerative Spinal Deformity. Neurosurgery 2015;77 Suppl 4:S75-91.

37. Le Huec JC, Saddiki R, Franke J, Rigal J, Aunoble S. Equilibrium of the human body and the gravity line: the basics. Eur Spine J 2011;20 Suppl 5:558-63.

38. Mac-Thiong JM, Roussouly P, Berthonnaud E, Guigui P. Sagittal parameters of global spinal balance: normative values from a prospective cohort of seven hundred nine Caucasian asymptomatic adults. Spine (Phila Pa 1976) 2010;35:E1193-8.

39. Shu S, Hu Z, Bao H, Shi J, Hu A, Grelat M, Liu Z, Sun X, Qian B, Cheng JCY, Lam TP, Chu WWC, Qiu Y, Zhu $Z$. An analysis of the interactions between the spine, pelvis, and lower limbs in asymptomatic adults with limited pelvic compensation. Quant Imaging Med Surg 2020;10:999-1007.

40. Gawri R, Moir J, Ouellet J, Beckman L, Steffen T, Roughley P, Haglund L. Physiological loading can restore the proteoglycan content in a model of early IVD degeneration. PLoS One 2014;9:e101233.

41. Sharma A, Parsons M, Pilgram T. Temporal interactions of degenerative changes in individual components of the lumbar intervertebral discs: a sequential magnetic resonance imaging study in patients less than 40 years of age. Spine (Phila Pa 1976) 2011;36:1794-800.

42. Siemionow K, An H, Masuda K, Andersson G, Cs-Szabo G. The effects of age, sex, ethnicity, and spinal level on the rate of intervertebral disc degeneration: a review of 1712 intervertebral discs. Spine (Phila Pa 1976) 2011;36:1333-9.

43. Pfirrmann CW, Metzdorf A, Elfering A, Hodler J, Boos N. Effect of aging and degeneration on disc volume and 
shape: A quantitative study in asymptomatic volunteers. J Orthop Res 2006;24:1086-94.

44. Takatalo J, Karppinen J, Niinimäki J, Taimela S, Näyhä S, Mutanen P, Sequeiros RB, Kyllönen E, Tervonen O. Does lumbar disc degeneration on magnetic resonance imaging associate with low back symptom severity in young Finnish adults? Spine (Phila Pa 1976) 2011;36:2180-9.

45. Zobel BB, Vadalà G, Del Vescovo R, Battisti S, Martina FM, Stellato L, Leoncini E, Borthakur A, Denaro V. T1 $\rho$ magnetic resonance imaging quantification of early lumbar intervertebral disc degeneration in healthy young adults. Spine (Phila Pa 1976) 2012;37:1224-30.

46. Maclean JJ, Lee CR, Alini M, Iatridis JC. Anabolic and catabolic mRNA levels of the intervertebral disc vary

Cite this article as: Menezes-Reis R, Garrido Salmon CE, Bonugli GP, Mazoroski D, Savarese LG, Herrero CFPS, Defino HLA, Nogueira-Barbosa MH. Association between spinal alignment and biochemical composition of lumbar intervertebral discs assessed by quantitative magnetic resonance imaging. Quant Imaging Med Surg 2021;11(6):2428-2441. doi: 10.21037/qims-20-40 with the magnitude and frequency of in vivo dynamic compression. J Orthop Res 2004;22:1193-200.

47. Battié MC, Videman T. Lumbar disc degeneration: epidemiology and genetics. J Bone Joint Surg Am 2006;88 Suppl 2:3-9.

48. Stone MA, Osei-Bordom DC, Inman RD, Sammon C, Wolber LE, Williams FM. Heritability of spinal curvature and its relationship to disc degeneration and bone mineral density in female adult twins. Eur Spine J 2015;24:2387-94.

49. Walter BA, Korecki CL, Purmessur D, Roughley PJ, Michalek AJ, Iatridis JC. Complex loading affects intervertebral disc mechanics and biology. Osteoarthritis Cartilage 2011;19:1011-8. 